\title{
Trained Innate Immunity: New Immunological Memory Mechanisms
}

\author{
Quan-Zhen Lv' ${ }^{1}$, Yuan-Ying Jiang ${ }^{1}$, Hua Zhong ${ }^{1}$, Yu-Lin Qin² ${ }^{2}$ Zhong-Lan Yuan ${ }^{1}$ and Yan Wang*1 \\ ${ }^{1}$ School of Pharmacy, Second Military Medical University, China
}

${ }^{2}$ Department of Pharmacy, Minhang Hospital, Fudan University, China

Received: May 20, 2018; Published: May 29, 2018

*Corresponding author: Yan Wang, School of Pharmacy, Second Military Medical University, Shanghai 200433, China

\begin{abstract}
Trained innate immunity attracts more and more attention in recent years. Innate immune memory is observed in natural killer cells, monocytes and macrophages after being trained by certain antigens and non-specific strong immune responses may occur after the secondary stimulation. Mechanism studies reveal that induction of trained innate immunity depends on epigenetic reprogramming. In this article, the phenomenon, mechanisms and possible applications of the trained innate immunity are reviewed.
\end{abstract}

Keywords: Trained Innate Immunity; Bacillus Calmette-Guerin; $\beta$-Glucan; Epigenetic Reprogramming

Abbreviations: NK cells: Natural Killer cells; BCG: Bacillus Calmette-Guerin; HSV: Herpes Simplex Virus; LPS: Lipopolysaccharide; MDP: Muramyl Dipeptide; NOD2: Nucleotide Binding Oligomerization Domain Containing 2; Raf-1: Raf-1 Proto-Oncogene Serine/Threonine Kinase; RIP2: Receptor-Interacting Protein Kinase; cAMP: Cyclic Adenosine Monophosphate; PKA: Protein Kinase A; mTOR: Mammalian Target of Rapamycin complex

\section{Introduction}

Innate immune cells can be trained by certain vaccines, pathogens or antigens and exhibit strong non-specific immune responses upon secondary infections, which is similar to the memory of acquired immunity [1]. This phenomenon is called trained innate immunity. A character of trained innate immunity is significantly more cytokines production after the secondary stimulation, single or multiple pathogens, on trained innate cells [2].

\section{The Trained Innate Immune Cells and Antigens}

presently, studies on the trained innate immunity mainly focuse on NK cells, monocytes and macrophages [3]. The trained NK cells induced by BCG showed protective effects on the following systemic Candida albicans infection after 1 week [1]. In addition to the stimulation of pathogens or vaccines, the memory of NK cells could also be trained by cytokine combinations including IL-12, IL-15 and IL-18 $[4,5]$. Besides NK cells, macrophages or monocytes could be trained by various fungi, bacteria, virus or purified legends [6]. BCG trained monocytes and macrophages play important roles in non-specific protections against infections caused by C. albicans [7,8], Staphy lococcus aureus, Salmonella enteritidis, Mycobacterium fortuitum, Yersinia pestis, Klebsiella pneumoniae, Schistosoma mansoni, HSV, vaccinia virus, and Leishmania major in mice [9]. Moreover, mac rophages trained by $C$. albicans could protect mice against reinfections by not only virulent $C$. albicans but also Gram-positive bacteria S. aureus [10]. Monocytes trained by Saccharomyces cerevisiae or $S$. cerevisiae-derived chitin exhibited enhanced ability to kill live C. albicans, S. aureus, and Escherichia coli in contrast to non-trained control cells [11].

In recent years, more and more researchers have focused their interest on antigens that could induce trained innate immunity. Besides BCG and $\beta$-glucan, two widely studied antigens, many other antigens have also been reported to induce trained innate immunity [12], such as S. cerevisiae, S. cerevisiae-derived chitin, LPS of low dose, Plasmodium falciparum [13], vaccinia virus vaccine [14], measles vaccine [15] and TLR9 legend CpG ODN [16]. Collectively, antigens that could induce trained innate immunity include bacteria, fungi and viruses. We believe that more antigens can be found in the future.

\section{The Mechanisms of Training on Innate Immune Cells}

The trained innate immunity was originally studied in plants, which are lack of adaptive immune responses. The immune responses of plants were divided into systemic acquired resistance 
(SAR) and induced systemic resistance (ISR) according to different inducing factors and pathways. When secondary infections occurred in plants, both SAR and ISR could produce strong non-specific immune responses to the pathogens, which were persistent or even permanent [17]. Recent evidence showed that the SAR of plants was mainly regulated by chromatin remodeling and epigenetic modifications. Those immune mechanisms of plants provided important insights into the molecular mechanisms of trained immunity in mammalians.

Some research progress has been made on trained immunity in mammalians. Mice vaccinated with BCG showed enhanced pro-inflammatory cytokine production in response to secondary pathogenic infections, and that was attributed to the trained immunity of NK cells and monocytes. The BCG-induced trained immunity depended on phagocytosis and the recognition of MDP through intracellular NOD2 receptors. Through NOD2 receptors, Rip2 kinase was activated and further the activated kinase promoted an epigenetic reprogramming through histone tri-methylation at H3K4 (H3K4me3) (Figure 1) [18]. The epigenetic programming induced by BCG in vivo could persist for more than one year [19]. In addition to the BCG-induced H3K4me3, training induced by C. albicans or $\beta$-glucan was also associated with epigenetic modifications, particular stable changes in histone tri-methylation at H3K4 and histone acetylation at H3K27. Further mechanism studies identified two crucial signal pathway in the trained immunity induced by $\mathrm{C}$. albicans or $\beta$-glucan. One was the non-canonical Raf-1-dependent pathway, which can be activated by C-type lectin receptor Dectin-1. The other was the cAMP-PKA-dependent signal pathway. Besides, trained innate cells exhibited great changes in the cellular metabolisms, as the genes involved in glucose and amino acids metabolisms were unregulated [10]. More specifically, training with $\beta$-glucan resulted in a Dectin-1/Akt/mTOR/HIF-1 $\alpha$ pathway activation that switched cellular metabolism from oxidative phosphorylation to glycolysis (Figure 1) [20]. Trained by C. albicans or $\beta$-glucan, murine monocytes or macrophages became more capable of producing inflammatory cytokines, as well as improved ability of phagocytosis and killing pathogens [21].

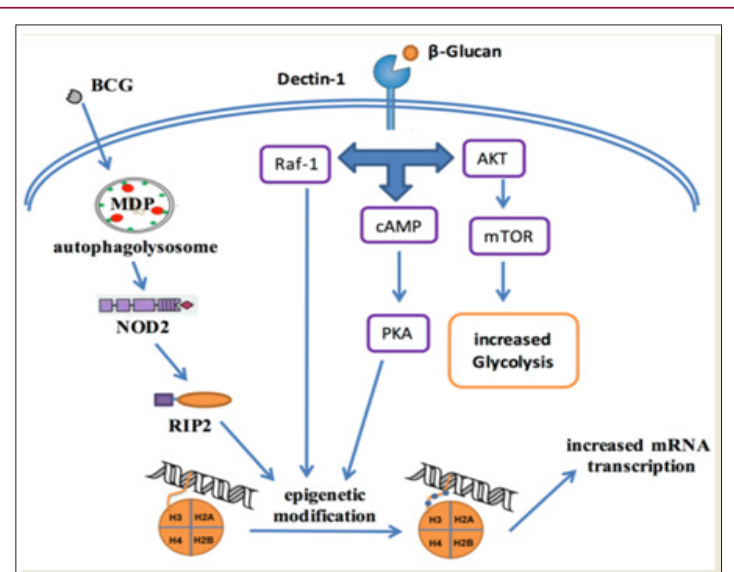

Figure 1: Cellular signaling pathways involved in the training of innate immune cells. Signaling pathways triggered by different receptors lead to epigenetic modifications of histone in the nuclear, resulting in increased mRNA transcription and cytokines.

\section{Conclusion}

The reveal of trained innate immunity has updated our understanding of immune memory. In the NK cells, monocytes and macrophages, after training by various antigens, epigenetic changes/ modifications and rewiring of gene transcription occurred, which may lead to immune memory. This feature of innate cells could bring benefits to the host, such as protection against infectious pathogens or reversion of some tumor processes. Discovering new immune adjuvants based on the theory of trained immunity is a promising strategy to conquer infectious diseases [22]. Nevertheless, further studies concerning trained immunity in different diseases are still needed [23], which will benefit the discovery of new therapeutic agents?

\section{Acknowledgement}

This work was supported by the National Natural Science Foundation of China (81772124)

\section{References}

1. Quintin J, Cheng SC, van der Meer JW, Netea MG (2014) Innate immune memory: towards a better understanding of host defense mechanisms. Curr Opin Immunol 29: 1-7.

2. Netea MG, van der Meer JW (2017) Trained Immunity: An Ancient Way of Remembering. Cell Host Microbe 21(3): 297-300.

3. Netea MG, Joosten LA, Latz E, Mills KH, Natoli G, et al. (2016) Trained immunity: A program of innate immune memory in health and disease. Science 352(6284): aaf1098.

4. Cooper MA, Elliott JM, Keyel PA, Yang L, Carrero JA, et al. (2009) Cytokineinduced memory-like natural killer cells. Proc Natl Acad Sci USA 106(6): 1915-1919.

5. Romee R, Schneider SE, Leong JW, Chase JM, Keppel CR, et al. (2012) Cytokine activation induces human memory-like NK cells. Blood 120(24): 4751-4760.

6. van der Meer JW, Joosten LA, Riksen N, Netea MG (2015) Trained immunity: A smart way to enhance innate immune defence. Mol Immunol 68(1): 40-44.

7. van 't Wout JW, Poell R, van Furth R (1992) The role of BCG/PPDactivated macrophages in resistance against systemic candidiasis in mice. Scand J Immunol 36(5): 713-719.

8. Smith SG, Kleinnijenhuis J, Netea MG, Dockrell HM (2017) Whole Blood Profiling of Bacillus Calmette-Guerin-Induced Trained Innate Immunity in Infants Identifies Epidermal Growth Factor, IL-6, Platelet-Derived Growth Factor-AB/BB, and Natural Killer Cell Activation. Front Immunol 8: 644 .

9. Blok BA, Arts RJ, van Crevel R, Benn CS, Netea MG (2015) Trained innate immunity as underlying mechanism for the long-term, nonspecific effects of vaccines. J Leukoc Biol 98(3): 347-356.

10. Quintin J, Saeed S, Martens JHA, Giamarellos-Bourboulis EJ, Ifrim DC, et al. (2012) Candida albicans infection affords protection against reinfection via functional reprogramming of monocytes. Cell Host Microbe 12(2): 223-232.

11. Rizzetto L, Ifrim DC, Moretti S, Tocci N, Cheng SC, et al. (2016) Fungal Chitin Induces Trained Immunity in Human Monocytes during Crosstalk of the Host with Saccharomyces cerevisiae. J Biol Chem 291(15): 7961-7972.

12. Rusek P, Wala M, Druszczynska M, Fol M (2018) Infectious Agents as Stimuli of Trained Innate Immunity. Int J Mol Sci 19(2).

13. McCall MB, Netea MG, Hermsen CC, Jansen T, Jacobs L, et al. (2007) Plasmodium falciparum infection causes proinflammatory priming of human TLR responses. J Immunol 179(1): 162-171. 
14. Mayr A (2004) Taking advantage of the positive side-effects of smallpox vaccination. J Vet Med B Infect Dis Vet Public Health 51(5): 199-201.

15. Aaby P, Martins CL, Garly ML, Bale C, Andersen A, et al. (2010) Nonspecific effects of standard measles vaccine at 4.5 and 9 months of age on childhood mortality: randomised controlled trial. BMJ 341: c6495.

16. Verthelyi D, Gursel M, Kenney RT, Lifson JD, Liu S, et al. (2003) CpG oligodeoxynucleotides protect normal and SIV-infected macaques from Leishmania infection. J Immunol 170(9): 4717-4723.

17. Pieterse CM, Leon-Reyes A, Van der Ent S, Van Wees SC (2009) Networking by small-molecule hormones in plant immunity. Nat Chem Biol 5(5): 308-316.

18. Kleinnijenhuis J, Quintin J, Preijers F, Joosten LA, Ifrim DC, et al. (2012) Bacille Calmette-Guerin induces NOD2-dependent nonspecific protection from reinfection via epigenetic reprogramming of monocytes. Proc Natl Acad Sci U S A 109(43): 17537-17542.
19. Kleinnijenhuis J, Quintin J, Preijers F, Benn CS, Joosten LA, et al. (2014) Long-lasting effects of BCG vaccination on both heterologous Th1/Th17 responses and innate trained immunity. J Innate Immun 6(2): 152-158.

20. Cheng SC, Quintin J, Cramer RA, Shepardson KM, Saeed S, et al. (2014) mTOR- and HIF-1alpha-mediated aerobic glycolysis as metabolic basis for trained immunity. Science 345(6204): 1250684.

21. Garcia-Valtanen P, Guzman-Genuino RM, Williams DL, Hayball JD, Diener KR1 (2017) Evaluation of trained immunity by beta-1, 3 (d)-glucan on murine monocytes in vitro and duration of response in vivo. Immunol Cell Biol 95(7): 601-610.

22. Topfer E, Boraschi D, Italiani P (2015) Innate Immune Memory: The Latest Frontier of Adjuvanticity. J Immunol Res 2015: 478408.

23. Arts RJW, Joosten LAB, Netea MG (2018) The Potential Role of Trained Immunity in Autoimmune and Autoinflammatory Disorders. Front Immunol 9: 298.

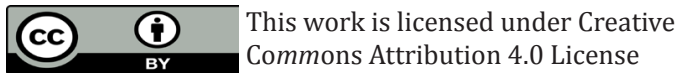

Submission Link: https://biomedres.us/submit-manuscript.php

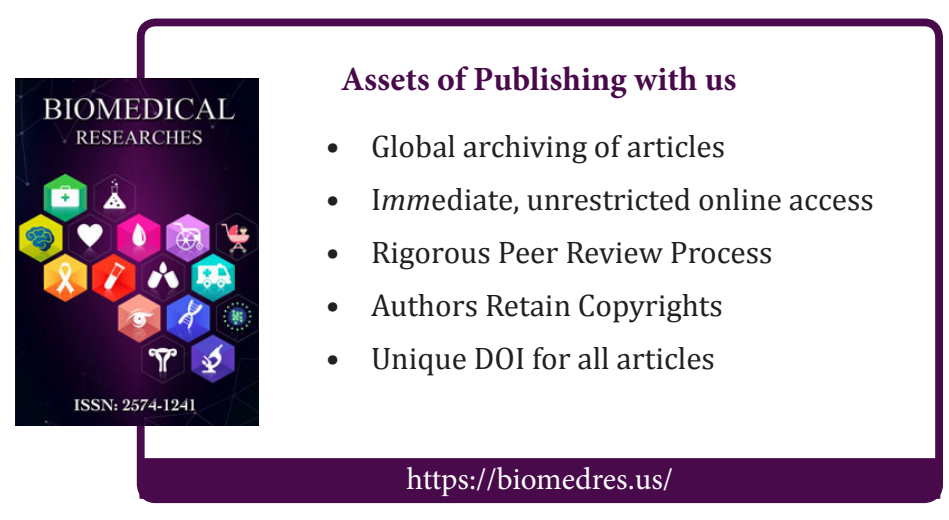

